\title{
Strong Coupling of Epsilon-Near-Zero Phonon Polaritons in Polar Dielectric Heterostructures
}

\author{
Nikolai Christian Passler, ${ }^{* \dagger}$ Christopher R. Gubbin ${ }^{\ddagger}{ }^{\ddagger}$ Thomas Graeme Folland, \\ Ilya Razdolski, ${ }^{\dagger}$ D. Scott Katzer, ${ }^{\S}$ David F. Storm, ${ }^{\S}$ Martin Wolf, ${ }^{\dagger}$ Simone De \\ Liberato, ${ }^{\ddagger}$ Joshua D. Caldwell,,$\S$ and Alexander Paarmann ${ }^{*, \dagger}$ \\ $\dagger$ Fritz-Haber-Institut der Max-Planck-Gesellschaft, Faradayweg 4-6,14195 Berlin, Germany \\ $\ddagger$ School of Physics and Astronomy, University of Southampton, Southampton SO17 1BJ, \\ United Kingdom \\ ฯ Vanderbilt Institute of Nanoscale Science and Engineering, 2301 Vanderbilt Place, PMB \\ 350106, Nashville, TN 37235-0106, USA \\ $\S U S$ Naval Research Laboratory, 4555 Overlook Avenue SW, Washington DC 20375, USA \\ E-mail: passler@fhi-berlin.mpg.de; alexander.paarmann@fhi-berlin.mpg.de
}

\section{Abstract}

We report the first observation of epsilon near zero (ENZ) phonon polaritons in an ultrathin AlN film fully hybridized with surface phonon polaritons ( $\mathrm{SPhP}$ ) supported by the adjacent $\mathrm{SiC}$ substrate. Employing a Hopfield strong coupling model for the analysis of the dispersion and electric field distribution in these hybridized modes, we show that they share the most prominent features of the two precursor modes. The novel ENZ-SPhP coupled polaritons with a highly propagative character and deeply sub-wavelength light confinement can be utilized as building blocks for future infrared and terahertz $(\mathrm{THz})$ nanophotonic integration and communication devices.

\section{Keywords}

surface phonon polariton, epsilon near zero, infrared, nanophotonics, polar crystal, strong coupling, hybridization

Integrated $\mathrm{THz}$ photonics relies on the development of artificially designed nano-scale metamaterials, where subwavelength structures in periodic patterns enable precise tuning of the material's optical response. ${ }^{1-3}$ Truly extraordinary light propagation characteristics can be achieved in metamaterial-based epsilon near zero (ENZ) media, ${ }^{4,5}$ i.e. where the dielectric permittivity is vanishingly small. In particular, remarkable properties of the ENZ photonic modes include tunneling through narrow distorted channels, ${ }^{6,7}$ enhanced nonlinearoptical conversion efficiency via enforced phasematching, ${ }^{8-10}$ provide high emission directionality $^{11-13}$ and enable polaritonic waveguiding modes with broken time inversion symmetry and reduced scattering rate. ${ }^{14,15}$ Commonly, these effects are studied for bulk ENZ photonic modes excited in carefully designed metamaterials based on plasmonic nanostructures, which are usually characterized by high losses. ${ }^{16,17}$

A complimentary approach aims at utilizing the naturally occuring zero-crossing of the dielectric function in the spectral vicinity of intrinsic material vibrations, such as the transverse optical (TO) and longitudinal optical (LO) phonons in polar dielectric crystals. ${ }^{18-20}$ Simultaneously, these materials can support surface phonon polaritons (SPhPs) in the reststrahlen band, which is bounded by these two phonon frequencies $\omega_{T O}$ and $\omega_{L O},{ }^{21,22}$ offering the appealing possibility to obtain ENZ po- 
laritonic modes that could be employed as an ideal information carrier enabling effective longrange nanophotonic communication.

An important challenge of future nanophotonics is to find an appropriate class of systems where these ENZ polaritons can be efficiently excited (without significant losses) and coupled to other photonic excitations, while maintaining highly propagative character with reasonable group velocities. Employing freestanding films of polar dielectrics, ${ }^{19}$ however, bears little practical importance, and the low dispersion results in a non-propagative character of the ENZ polaritonic modes. ${ }^{18}$ In this work, we demonstrate the hybridization of an ENZ polariton and a propagating low-loss $\mathrm{SPhP}$ at an adjacent interface in the strong coupling regime, thus creating a new pair of coupled nanophotonic excitations to an evergrowing suite. ${ }^{23-27}$ We analyze the coupling of the ENZ and SPhP modes in an AlN/SiC bilayer, where the ENZ polariton in AlN occurs within the reststrahlen band of SiC. The novel coupled ENZ-SPhP modes inherit their properties from both ENZ and SPhP components, thus enabling highly efficient phase-matched exitation. Offering broad functionality, these ENZ-SPhP coupled modes feature a unique combination of deeply subwavelength confinement, large enhancements of the local electromagnetic fields as well as an intrinsically low-loss, propagative character with non-zero group velocity.

A SPhP mode supported at the interface of a polar dielectric in the Reststrahlen band is split into two branches upon reducing the film thickness $d$ (Fig. 1a), known as symmetric and antisymmetric modes. ${ }^{18,19,28}$ Remarkably, in ultrathin films $\left(d / \lambda \sim 10^{-2}\right.$, with $\lambda$ being the free-space wavelength) the upper (symmetric) mode is pushed towards the longitudinal optical (LO) phonon frequency, ${ }^{18}$ where the real part of the dielectric permittivity approaches zero $\left(\varepsilon^{\prime}\left(\omega=\omega_{\mathrm{LO}}\right)=0\right)$. While this ultrathin film ENZ polariton loses its dispersive character, its ultra-long wavelength leads to a strongly subwavelength mode confinement, enabling a gigantic enhancement of the electric field with minimal phase change over several times the free-space wavelength. The polariton disper-
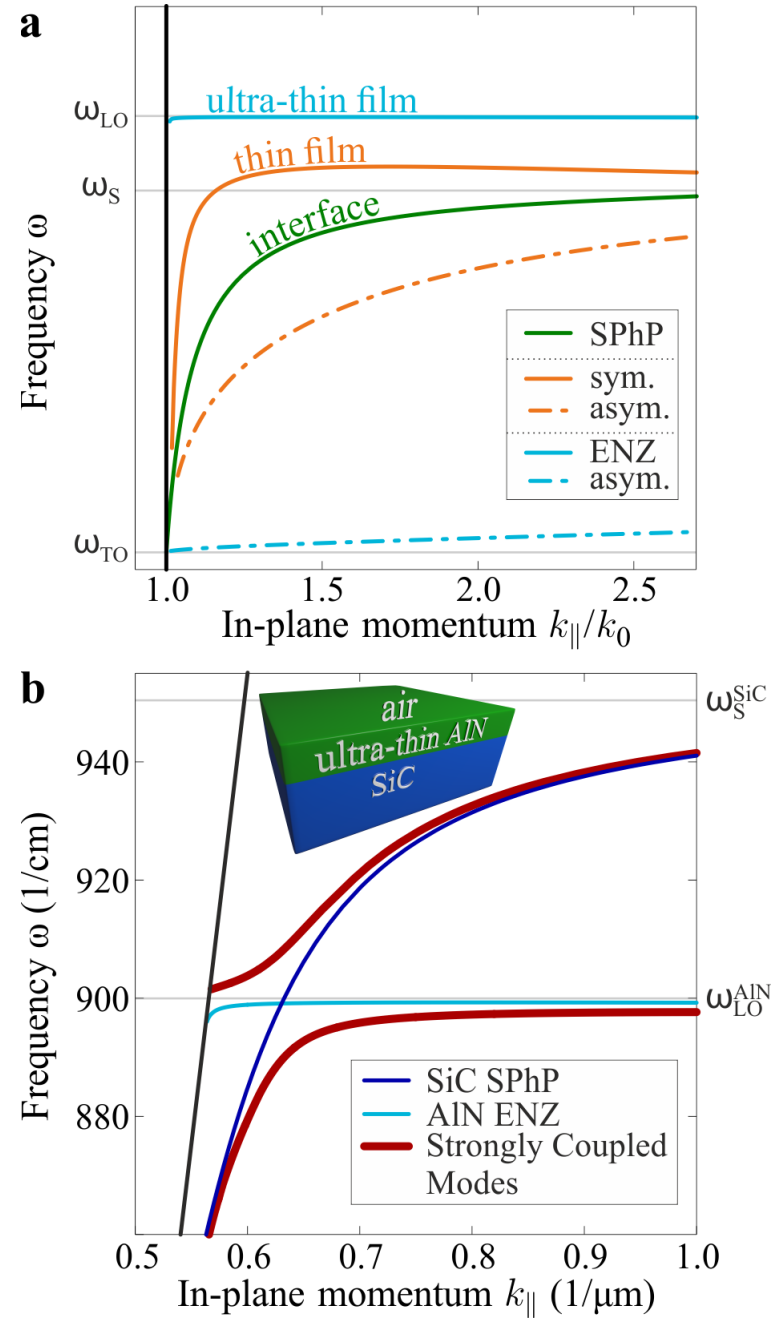

Figure 1: Interaction of a surface phonon polariton with an epsilon-nearzero mode. a A SPhP propagating at a single interface of air and a polar crystal has a dispersion relation (green curve) ranging from the transversal optical phonon frequency $\omega_{T O}$ up to a cut-off frequency $\omega_{S}$, shown in green. For film thicknesses $d<\lambda / 2$, where $\lambda$ is the free-space wavelength, a symmetric and an antisymmetric branch appear (orange), splitting further apart with decreasing $d$. In the ultrathin limit of $d<\lambda / 50$, the upper branch is pushed close to the longitudinal optical phonon frequency $\omega_{L O}$ (light blue), where it is termed an epsilon-near-zero mode. b When an ultrathin AlN film is placed on bulk $\mathrm{SiC}$, the AlN epsilon-near-zero mode (light blue) intersects the $\mathrm{SiC} \mathrm{SPhP}$ dispersion relation (dark blue). These modes strongly interact, inducing an avoided crossing and forming two new dispersion branches of the coupled system, drawn in red. 
sion curves in the bilayer with an ultrathin AlN film on top of a $\mathrm{SiC}$ substrate are exemplified in Fig. 1b. Individually, the AlN film exhibits a non-dispersive ENZ polariton mode (light blue) and the SiC a SPhP (dark blue). The combined bilayer structure (inset Fig. 1b), however, reveals a strong interaction between the two modes, leading to two new dispersive branches featuring an avoided crossing (red).

In the experiments, we realize phase-matched excitation of the coupled modes by employing the Otto prism geometry, ${ }^{29,30}$ with a schematic provided in Fig. 2a. For total internal reflection inside the prism, the evanescent wave at the prism backside enables phase-matched excitation of the polariton modes in the sample. Spectroscopic reflectivity measurements with varied incidence angle allow for the mapping out of the polariton dispersion relation (for details on the experimental methods see Supplementary Information Section 1). In our AlN/SiC structure, two polariton branches are present, and hence two resonance dips can be observed (Fig. $2 \mathrm{~b}$ ), showing that the frequency splitting of the branches increases for thicker films. This trend is also evident in the experimental reflectivity maps and the transfer matrix calculations in Fig. 3. Furthermore, our data reveal the anticipated avoided crossing in the dispersion of the interacting polariton modes, corroborating the strong coupling mechanism.

Analytically, the strong coupling can be described by a system of two coupled oscillators, modeling the ENZ and the SPhP modes respectively. This allows us to calculate, in an analytically transparent way, the dispersion and the in-plane $E$-field distributions of the normal modes in the hybrid structure. To this end, we use a Hopfield model analogous to that commonly employed in solid-state cavity quantum electrodynamics, although we emphasize that in the present work we limit ourselves to studying only classical phenomena correctly reproduced by a transfer matrix approach. In the rotating wave approximation, the two-oscillator Hopfield Hamiltonian for our system takes the $\mathbf{a}$
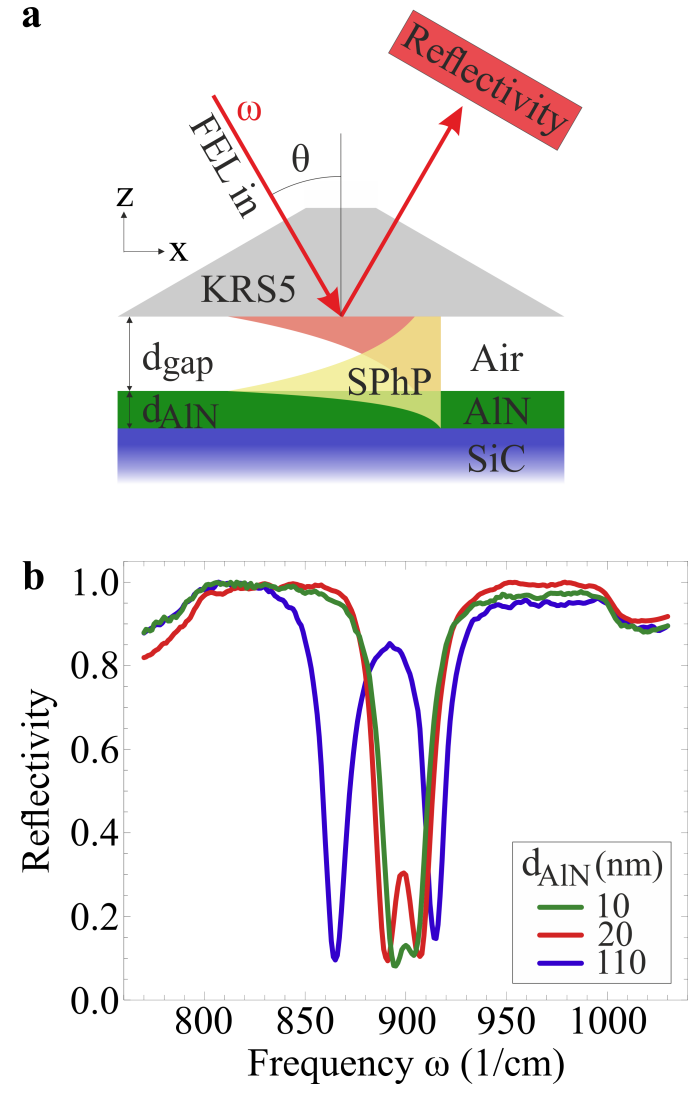

Figure 2: The Rabi splitting dependence on the AlN film thickness. a Prism coupling setup implementing the Otto configuration, where a highly-refractive KRS5 prism $\left(n_{K R S 5} \approx 2.4\right)$ enables phase-matched excitation of phonon polaritons across a variable air gap. By tuning the incoming frequency $\omega$ and the incidence angle $\theta$, polariton dispersion curves can be mapped out. b Reflectivity spectra for three different AlN film thicknesses $d_{A l N}=10,20,110 \mathrm{~nm}\left(\theta=29,29,30^{\circ}\right.$ and $d_{\text {gap }}=4.8,4.5,3.7 \mu \mathrm{m}$, respectively). The resonance dips represent the two strongly interacting polariton branches in the $\mathrm{AlN} / \mathrm{SiC}$ heterostructure. 


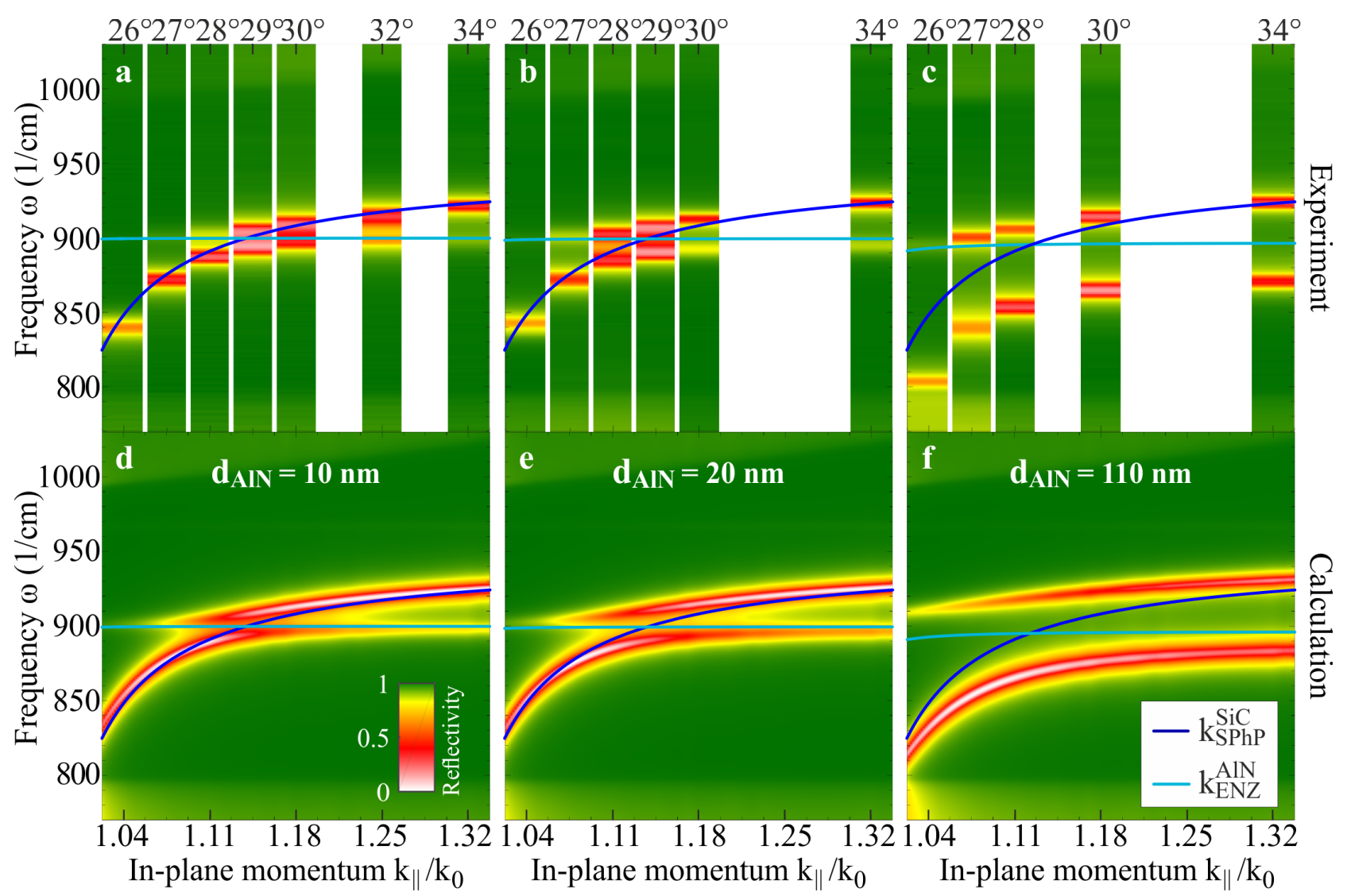

Figure 3: Mapping out the strongly coupled polariton dispersion. Experimental and calculated reflectivity maps for all three $d_{A l N}$, revealing the anticipated avoided crossing in the dispersion of the interacting polariton modes. The transfer matrix calculations (d-f) perfectly reproduce the experimental data (a-c). Clearly, the splitting of the modes is large for the thickest film, and decreases for smaller thicknesses. In the limit of a vanishing film thickness, the single continuous $\mathrm{SiC}$ $\mathrm{SPhP}$ dispersion curve would be recovered (dark blue line). The light blue line is the dispersion of the ultrathin film polariton in a freestanding AlN film.

form ${ }^{31-34}$

$\mathcal{H}=\sum_{q} \hbar \omega_{q}^{\mathrm{e}} \hat{a}_{q}^{\dagger} \hat{a}_{q}+\hbar \omega_{q}^{\mathrm{s}} \hat{b}_{q}^{\dagger} \hat{b}_{q}+\hbar g_{0}\left(\hat{a}_{q}^{\dagger} \hat{b}_{q}+\hat{a}_{q} \hat{b}_{q}^{\dagger}\right)$,

where $\hat{a}_{q}^{\dagger}\left(\hat{a}_{q}\right)$ and $\hat{b}_{q}^{\dagger}\left(\hat{b}_{q}\right)$ are the bosonic creation (annihilation) operators for the ENZ and SPhP modes, $\omega_{q}^{\mathrm{e}, \mathrm{s}}$ their frequencies, respectively, and $g_{0}$ is the Rabi splitting. The eigenfrequencies $\omega_{q}^{ \pm}$of the coupled system are then given by

$$
\omega_{q}^{ \pm}=\frac{\omega_{q}^{e}+\omega_{q}^{s} \pm \sqrt{\left(\omega_{q}^{e}-\omega_{q}^{s}\right)^{2}+4 g_{0}^{2}}}{2} .
$$

We employ Eq. 2 to calculate the dispersion analytically (Fig. 4a), finding excellent agreement of our strong coupling model with the numerical calculations and demonstrating that the description of the energetic hybridization of the modes in terms of strong coupling is correct. Moreover, the Rabi splitting $g_{0}$ in strong coupling systems scales as the square root of the number of oscillators, ${ }^{35-37}$ being proportional to the AlN film thickness. The Rabi splitting we extract using Eq. 2 follows this expected square root dependence, see Fig. 4b. This behavior is reproduced by the experimental coupling strengths also shown in Fig. 4b, determined by the frequency splitting in the respective reflectivity spectra, see Fig. $2 b$.

Furthermore, our simple analytical model also correctly describes the electric field profiles. This is illustrated in Fig. 4c, where we show the coinciding analytical and numerically calculated field intensities of both modes in front 


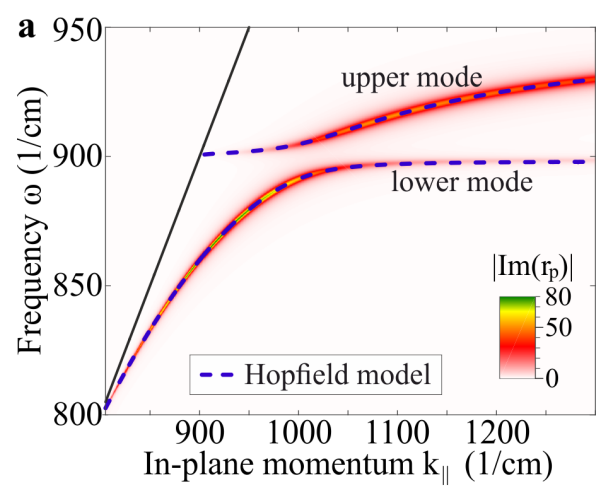

$\begin{array}{cccc}900 & 1000 & 1100 & 1200 \\ \text { In-plane momentum } & \mathrm{k}_{\|} & (1 / \mathrm{cm})\end{array}$
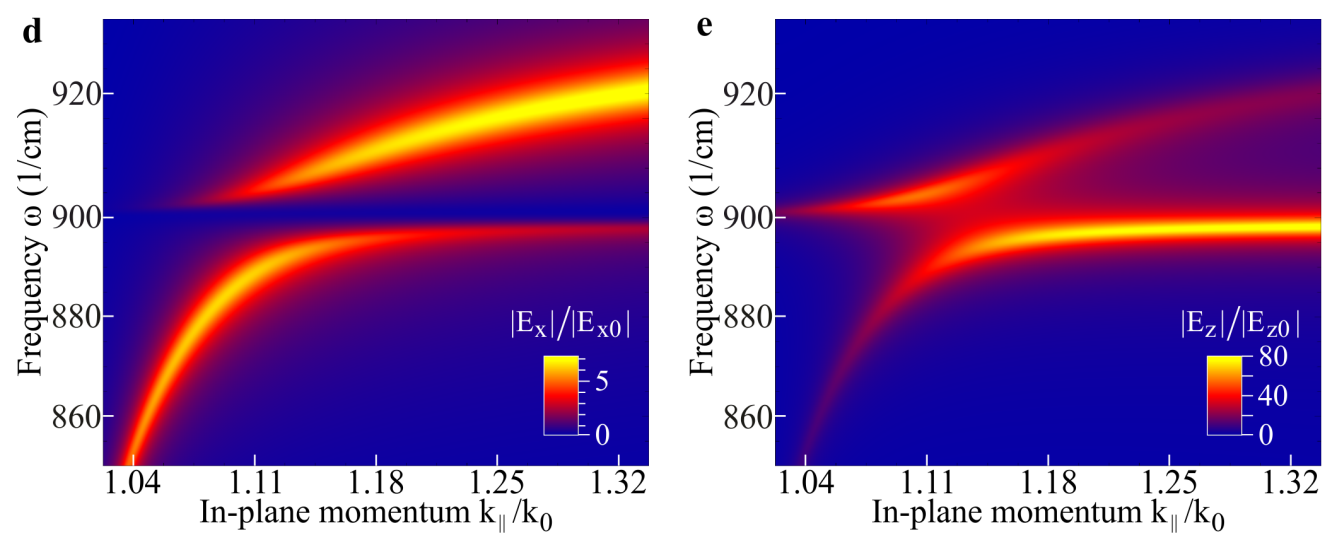
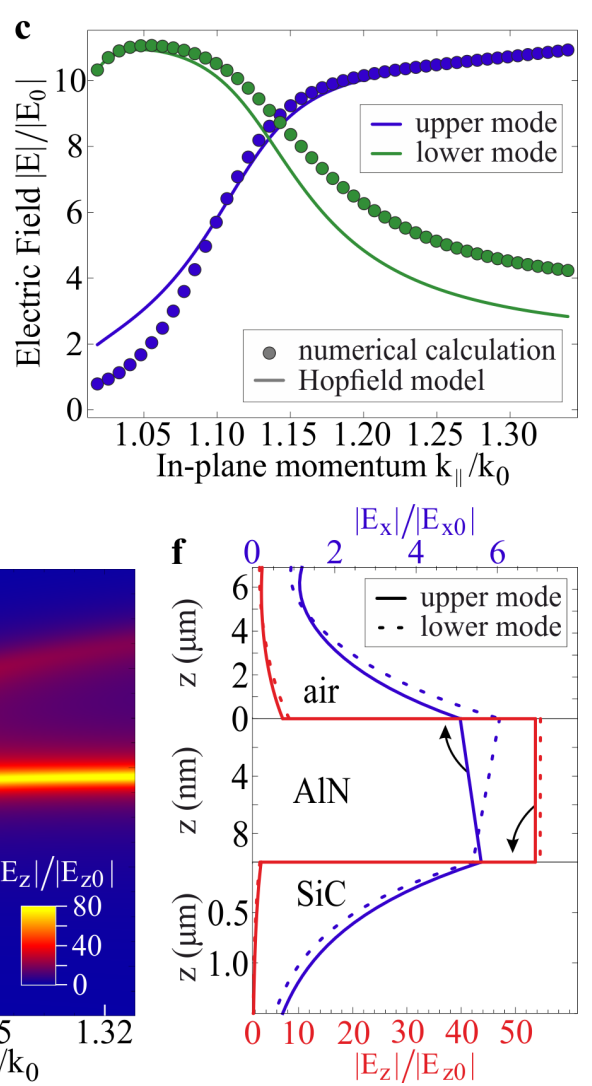

Figure 4: Coupling between the ENZ and SPhP modes. a Dispersion curves obtained by the analytical strong coupling model (dashed line) for an AlN film thickness of $d_{A l N}=10 \mathrm{~nm}$ on $\mathrm{SiC}$ on top of the numerically calculated dispersion curves obtained by computing the absolute imaginary part of the p-polarized reflection coefficient of the three layer system air/AlN/SiC. The analytical model features excellent agreement with the numerical calculations. b Rabi splitting $g_{0}$ as a function of $d_{A l N}$ extracted from the analytical model (green circles) and experiments (red diamonds). As can be seen from the simple model fit (solid line, $a=0.26 \cdot 10^{-13} \mathrm{~m}^{3}$ ), the Rabi splitting follows a square root function characteristic for strong coupling. c Electric field strength obtained by the analytical model (solid line) and numerical calculations (circles) for the two strongly coupled modes, calculated at a probe point in air at the sample surface. At the avoided crossing, the two modes are completely hybridized, sharing equal field strength. At smaller in-plane momentum than at the avoided crossing, the lower branch has a larger field strength than the upper branch, and vice versa at larger momenta. The mode with larger field strength exhibits SPhP character, since at the specific probe point, the SPhP field dominates, while the ENZ polariton is localized inside the AlN film. d,e Normalized in-plane $\left(E_{x}\right)$ and out-of-plane $\left(E_{z}\right)$ field components along the entire dispersion. The SPhP has large in-plane and the ENZ polariton large out-of-plane fields, while the respective other component is small. This allows to track the mode nature (SPhP or ENZ polariton) of both dispersion branches, which is exchanged at the avoided crossing, along the entire momentum range. f Normalized $E_{x}$ and $E_{z}$ fields of the upper and the lower mode at resonance $\left(k_{\|} / k_{0}=1.13\right)$ across the air/AlN/SiC structure. The layer thicknesses are not to scale with respect to each other. 
of the sample. We note that while a bare $\mathrm{SiC}$ substrate allows the SPhP component of the coupled modes to be quantified, the ENZ polariton component depends decisively on the substrate material and hence cannot be straightforwadly quantified. Therefore, we assumed the ENZ polariton to be fully confined in the AlN film, i.e., the field at the probe point is solely determined by the SPhP component of the coupled modes. This assumption is the reason for the discrepancy between the analytical and calculated field intensities at in-plane momenta where the respective mode features ENZ character $\left(k_{\|} / k_{0}<1.1\right.$ and $k_{\|} / k_{0}>1.2$ in Fig. 4c). However, despite its simplicity, our model reproduces the numerical field amplitudes extremely well, proving that the coupled modes can be described as a linear superposition of the ENZ and SPhP modes, weighted by the Hopfield coefficients. In consequence of this linear relationship, the strongly coupled modes at the avoided crossing share equal weights of SPhP and ENZ character, while the respective partitions change along the dispersion: the lower polariton starts as pure $\mathrm{SPhP}$ at small $k$ and switches to ENZ beyond the avoided crossing, while the upper polariton shows the opposite behavior.

This switching of the mode nature can be illustrated by means of the in-plane $\left(E_{x}\right)$ and out-of-plane $\left(E_{z}\right)$ electric field components inside the AlN film, shown in Fig. 4d and e, respectively. Note that the $\mathrm{SPhP}$ is characterized by a large in-plane field, whereas the ENZ polariton features pronounced out-of-plane field enhancement. The lower branch has strong inplane fields at lower momentum (Fig. 4d), illustrating that the mode is predominantly $\mathrm{SPhP}$ in nature. In contrast, the upper branch exhibits strong out-of-plane character at low $k_{\|}$(Fig. 4e). Across the strong coupling region, the modes interchange these characteristics, with the upper branch exhibiting strong in-plane and the lower out-of-plane fields. At the avoided crossing, the fields of both modes are apparent and of equal weight, and even the spatial $E_{x}$ and $E_{z}$ field distributions of the modes across the multilayer structure show high agreement (Fig. 4f). We have thus demonstrated the strong coupling and full hybridization of an ultrathin film ENZ phonon polariton with a SPhP in a polar dielectric heterostructure exemplified for $\mathrm{AlN} / \mathrm{SiC}$. However, we emphasize that strong coupling will emerge for a large number of hybrid systems that feature overlapping reststrahlen bands of the two constituents. ${ }^{21,22}$ Furthermore, strong coupling can also be observed in the inverse structure of an ultrathin SiC film on AlN, ocurring at the TO frequency of $\mathrm{SiC}$ (see Supplementary Information Section 3).

Notably, the observed strong coupling physics uniquely arises for ultrathin films with a deeply sub-wavelength thickness of $d \sim \lambda / 1000$ (11 nm for $\mathrm{AlN}$ ). Despite the mode splitting following the $\sqrt{d}$ dependence for up to $d \gtrsim 500 \mathrm{~nm}$, already for film thicknesses $d>50 \mathrm{~nm}$, the interaction of the modes is not well-described by the strong coupling model anymore. This deviation from Eq. 1 is observed for both the mode dispersion as well as the field profiles, see Supplementary Information Section 4. In contrast, within the strong coupling regime, thinner films provide higher quality ENZ wave characteristics, yet featuring full hybridization with the low-loss, highly confined SPhP, see Fig. 4a-c. Thereby, the ENZ-SPhP modes offer a new promising approach for $\mathrm{THz}$ photonics on the nanoscale using traditional materials like III-V and II-VI semiconductors. ${ }^{22}$ For instance, $\mathrm{PbSe} / \mathrm{PbS}$ core-shell nanostructures ${ }^{38}$ with wide tunability of the optical properties in the near-infrared additionally feature strongly coupled ENZ-SPhP modes in the $\mathrm{THz}$ range, allowing for a unique multispectral photonic integration.

To demonstrate that the general character of strong coupling is not restricted to the prismbased experiments, we complement our results with electromagnetic simulations of optical near-field scattering. Motivated by the published results on scattering type scanning near field optical microscopy (s-SNOM) of $\mathrm{Au}$ nanostructures on $\mathrm{SiC}$ and boron nitride, ${ }^{39,40}$ we consider here a $\mathrm{Au}$ cylinder on top of the AlN film on a SiC substrate, see Fig. 5a. In the simulations, we monitor the spatial distribution of the electric field induced by normally 

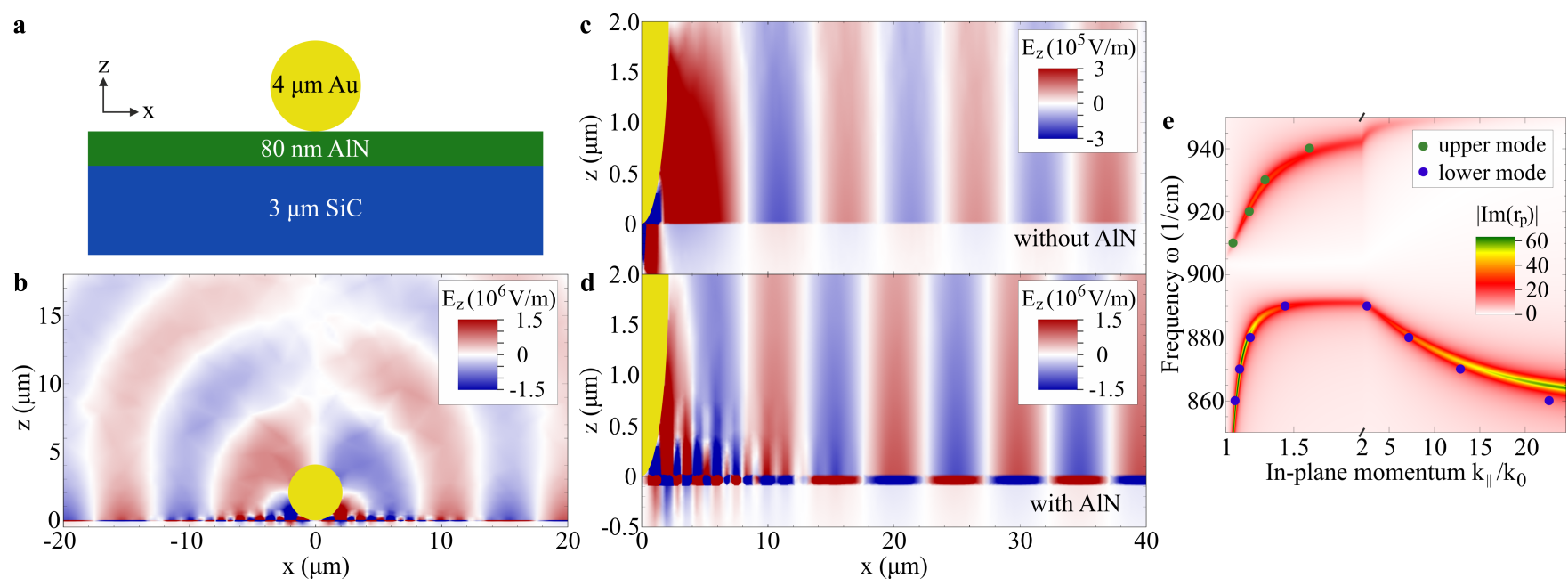

Figure 5: Numerical simulations of a high-k polariton with negative group velocity. a shows the simulation geometry, where plane waves are normally incident on a gold particle on the surface. Due to the large size of the particle, in $\mathbf{b}$ both surface waves and scattered free space waves are observed. c,d Surface electromagnetic field for a bare SiC substrate (c) and with an $80 \mathrm{~nm}$ AlN film on top (d). The field distributions in b-d were all calculated at an excitation frequency of $880 \mathrm{~cm}^{-1}$. e Comparison between the dispersion relation calculated by a transfer matrix approach (background color map) and the values extracted from the simulations (circles). At large in-plane momentum $\left(k_{\|} / k_{0}>2\right)$, the negative slope of the dispersion of the lower mode reveals its negative group velocity.

incident plane waves at a series of frequencies. Whilst the $80 \mathrm{~nm}$ AlN film was chosen in this simulation for a clear identification of the coupled polaritonic modes, similar results can be obtained for the AlN film thicknesses discussed earlier in this work.

Results for an $80 \mathrm{~nm}$ AlN film on top of a $\mathrm{SiC}$ substrate are presented in Fig. 5b, showing the full electromagnetic field scattered from the Au cylinder at $880 \mathrm{~cm}^{-1}$. To focus on the polaritons launched along the surface, in Fig. $5 \mathrm{c}$ and $\mathrm{d}$ we consider the spatial distribution of the electric field along the surface in the two cases, namely, with and without the AlN layer. Without the AlN film (Fig. 5c) the results of the simulations are indicative of a propagating $\mathrm{SPhP}$ wave launched across the surface with minimal confinement. In the presence of the AlN film, however, the simulations reveal two distinct modes with unequal wavelengths, see Fig. 5d. The longer wavelength mode corresponds to that observed in our experiments, which is slightly compressed when compared with the wave propagating on the $\mathrm{SiC}$ surface. The short wavelength mode, however, was not observed in our measurements due to its large in-plane momentum. We attribute this mode to the breakdown of the thin film approximation for ENZ behaviour which has been theoretically predicted at extremely large $\mathrm{k} .{ }^{18}$ One of the most striking features of this large-k mode is its negative group velocity, clearly visible from the dispersion in Fig. 5e and confirmed by the timedependent E-field distributions (Movie S1).

To better understand both the positive and negative group velocity modes observed in these simulations, we quantify the polariton wavelength by Fourier transform (FT) of the simulated electromagnetic field. Here, we take the FT of the normal projection of the complex $E_{z}$ field on the top surface of the AlN layer at a series of different excitation frequencies. The dispersion determined from the $E_{z}$ Fourier spectra are plotted and compared against transfer matrix simulations of the polariton dispersion in Fig. 5e. The excellent agreement between the numerically simulated frequencies and the calculated dispersion indicates that the key results of this paper should be observable by the s-SNOM technique. Additionally, the lat- 
ter should enable the observation of the high- $\mathrm{k}$ mode with negative dispersion, which is otherwise inaccessible in the prism-coupling experiments. We thus envisage rich perspectives of near-field microscopy in visualizing coupled phonon-polaritonic modes in hybrid or multilayer systems.

In conclusion, in this work we have demonstrated and characterized polaritonic modes in a strong coupling regime between an ENZ polariton and a bulk $\mathrm{SiC} \mathrm{SPhP}$ in an ultrathin $\mathrm{AlN} / \mathrm{SiC}$ structure. The full mode hybridization at the avoided crossing enables unique propagating ENZ polaritons. We have performed numerical simulations, revealing that the s-SNOM approach enables the observation of both the propagation length of the coupled ENZ-SPhP modes, and the properties of polaritons featuring negative group velocity. Our results illustrate the high suitability of near-field techniques like s-SNOM for the investigation of low-loss ENZ polaritons in polar dielectric heterostructures, in order to further establish their potential for nanophotonic applications. We envision the generalization of employing polar dielectric ENZ heterostructures to open up a new platform of deeply sub-wavelength integrated $\mathrm{THz}$ photonics based on strongly coupled ENZ-SPhPs.

\section{Methods}

\section{Experimental}

The substrate of our samples is hexagonal $6 \mathrm{H}$ $\mathrm{SiC}$ for the $110 \mathrm{~nm} \mathrm{AlN}$ film, and $4 \mathrm{H}-\mathrm{SiC}$ for the other two samples, all three with the extraordinary axis perpendicular to the sample (c-cut). The AlN layers were grown by RFplasma assisted molecular beam epitaxy, and therefore also exhibit a $c$-cut, hexagonal crystal structure.

As an excitation source, we employ a midinfrared free electron laser (FEL) with small bandwidth $(\sim 0.3 \%)$ and wide tunability of $3-50 \mu \mathrm{m}$, covering the spectral ranges of the $\mathrm{SiC}$ and $\mathrm{AlN}$ reststrahlen bands (details on the FEL have been reported elsewhere ${ }^{41}$ ). While the frequency is scanned by tuning the FEL, different in-plane momenta can be accessed via the incidence angle $\theta$ by rotating the entire Otto geometry (see Supplementary Information Section 1 for more details), thus allowing for mapping out the complete dispersion curves experimentally. ${ }^{30}$ In contrast to alternative approaches, the Otto geometry features experimental control over the excitation efficiency through tunability of the air gap width $d_{\text {gap }}$. At each incidence angle, spectra were taken at several $d_{\text {gap }}$. For the reconstruction of the dispersion curves (Fig. 3), we selected the spectra at a gap size of critical coupling conditions $d_{\text {crit }},{ }^{30}$ i.e. where the polariton is excited the most efficiently (Supplementary Information Section 2). Direct read-out of the gap width $d_{\text {gap }}$ with a range of $d=1-50 \mu \mathrm{m}$ is realized via whitelight interferometry, while the contrast of the interference spectrum grants parallel alignment of prism and sample.

\section{Theoretical}

\section{Transfer Matrix}

All calculations of the optical response and field distributions of Fig. 1-4 were performed using a generalized $4 \times 4$ transfer matrix formalism. ${ }^{42}$ In short, the formalism allows for the calculation of reflection and transmission coefficients in any number of stratified media with arbitrary dielectric tensor, which allows to account for the anisotropy of our samples.

\section{Three-Layer Dispersion}

The dispersion curves in Fig. 1 were obtained by numerical evaluation of the three-layer polariton dispersion formula ${ }^{18,28,43}$

$$
1+\frac{\varepsilon_{1} k_{z 3}}{\varepsilon_{3} k_{z 1}}=i \tan \left(k_{z 2} d\right)\left(\frac{\varepsilon_{2} k_{z 3}}{\epsilon_{3} k_{z 2}}+\frac{\varepsilon_{1} k_{z 2}}{\varepsilon_{2} k_{z 1}}\right),
$$

where the subscripts $i=1,2,3$ correspond to the three stacked media, $\varepsilon$ is the dielectric function, $d$ the film thickness of material 2 , $k_{z i}=\sqrt{\frac{\omega}{c} \varepsilon_{i}-k_{\|}}$the out-of-plane momentum, 
and $k_{\|}$the in-plane momentum conserved in all layers.

\section{Hopfield Model}

The Rabi splitting $g_{0}$ of the strong coupling model in Eq. 1 is introduced as a phenomenological coupling parameter, and is equivalent to the overlap of the substrate SPhP and ENZ polariton in a classical electromagnetic approach. The eigenfrequencies (Eq. 2) of the coupled system are found by diagonalization of the Hopfield-Bogoliubov matrix $H_{q}{ }^{33}$

$$
H_{q}=\left(\begin{array}{cc}
\omega_{q}^{e} & g_{0} \\
g_{0} & \omega_{q}^{s}
\end{array}\right)
$$

for each in-plane wavevector $q$ individually, where $e$ and $s$ stands for the ENZ polariton and the substrate $\mathrm{SPhP}$, respectively. The eigenvalues of these matrices yield the eigenfrequencies $\omega_{q}^{ \pm}$shown in Eq. 2, and the respective normalized eigenvectors $\left(X_{q}, Y_{q}\right)$ are built from the Hopfield coefficients $X_{q}$ and $Y_{q}$, describing the weighting factors of the ENZ polariton and the substrate SPhP which compose the two hybridized modes along the avoided crossing. The bosonic annihilation operators $\hat{p}_{q}$ of the coupled modes are then given by

$$
\begin{aligned}
& \hat{p}_{q}^{+}=X_{q} \hat{a}_{q}+Y_{q} \hat{b}_{q} \\
& \hat{p}_{q}^{-}=Y_{q} \hat{a}_{q}-X_{q} \hat{b}_{q},
\end{aligned}
$$

where the superscripts + and - denote the upper and the lower coupled polariton branch, respectively.

\section{CST Simulations}

Simulations for Fig. 5 were performed in CST studio suite ${ }^{44}$ using the frequency domain solver. To approximate the structure shown in Fig. 5a within a finite 3D model, a unit cell with a size of $250 \mu \mathrm{m}$ by $0.6 \mu \mathrm{m}$ was chosen, which minimized nearest neighbour interactions. The optical constants of the respective materials were taken from literature. ${ }^{45,46}$ Unit cell boundaries were used at the edges of the substrate, and a matched impedance layer was used to suppress substrate reflections. Fourier analysis was performed using a one dimensional field profile running along the top surface of the ENZ film down the center of the unit cell.

Acknowledgement We thank Wieland Schöllkopf and Sandy Gewinner for operating the FEL. D.S.K., D.F.S, and J.D.C. were supported by the Office of Naval Research through the U.S. Naval Research Laboratory and administered by the NRL Nanoscience Institute. The NRL team acknowledges the AlN characterization and processing contributions of Neeraj Nepal, Brian P. Downey and Neil P. Green. S.D.L. is a Royal Society Research Fellow and he acknowledges support from EPSRC Grant No. EP/M003183/1.

\section{Supporting Information Avail- able}

The following files are available free of charge.

Experimental details (Section 1), critical coupling conditions of the strongly coupled modes (Section 2), strongly interacting modes in materials with overlapping reststrahlen bands (Section 3), deviation from strong coupling for larger film thicknesses (Section 4), and simulated time-dependent E-field distributions (Movie S1).

\section{References}

(1) Joannopoulos, J. D.; Johnson, S. G.; Winn, J. N.; Meade, R. D. Photonic Crystals: Molding the Flow of Light; Princeton University Press, 2008.

(2) Burgos, S. P.; De Waele, R.; Polman, A.; Atwater, H. A. Nature Materials 2010, 9, 407-412.

(3) Degl'Innocenti, R.; Kindness, S. J.; Beere, H. E.; Ritchie, D. A. Nanophotonics 2018, 7, 127-144.

(4) Li, Y.; Kita, S.; Muñoz, P.; Reshef, O.; Vulis, D. I.; Yin, M.; Lončar, M.; Mazur, E. Nature Photonics 2015, 9, 738 742. 
(5) Liberal, I.; Engheta, N. Nature Photonics 2017, 11, 149-158.

(6) Silveirinha, M. G.; Engheta, N. Physical Review B - Condensed Matter and Materials Physics 2007, 76, 1-17.

(7) Edwards, B.; Al, A.; Silveirinha, M. G.; Engheta, N. Journal of Applied Physics 2009, 105.

(8) Argyropoulos, C.; Chen, P. Y.; D’Aguanno, G.; Engheta, N.; Alù, A. Physical Review B - Condensed Matter and Materials Physics 2012, 85, 1-5.

(9) Suchowski, H.; O’Brien, K.; Wong, Z. J.; Salandrino, A.; Yin, X.; Zhang, X. Science 2013, 342, 1223-1226.

(10) Mattiucci, N.; Bloemer, M. J.; D'Aguanno, G. Optics Express 2014, 22, 6381.

(11) Enoch, S.; Tayeb, G.; Sabouroux, P.; Guérin, N.; Vincent, P. Physical Review Letters 2002, 89, 213902.

(12) Ziolkowski, R. W. Physical Review E Statistical, Nonlinear, and Soft Matter Physics 2004, 70, 1-12.

(13) Kim, J.; Dutta, A.; Naik, G. V.; Giles, A. J.; Bezares, F. J.; Ellis, C. T.; Tischler, J. G.; Mahmoud, A. M.; Caglayan, H.; Glembocki, O. J.; Kildishev, A. V.; Caldwell, J. D.; Boltasseva, A.; Engheta, N. Optica 2016, 3, 339.

(14) Liu, R.; Roberts, C. M.; Zhong, Y.; Podolskiy, V. A.; Wasserman, D. ACS Photonics 2016, 3, 1045-1052.

(15) Engheta, N. Science 2007, 317, 1698 1702 .

(16) Drachev, V. P.; Chettiar, U. K.; Kildishev, A. V.; Yuan, H.-K.; Cai, W.; Shalaev, V. M. Optics Express 2008, 16, 1186-1195.

(17) Khurgin, J. B.; Boltasseva, A. MRS Bulletin 2012, 3\%, 768-779.
(18) Campione, S.; Brener, I.; Marquier, F. Physical Review B 2015, 91, 121408.

(19) Nordin, L.; Dominguez, O.; Roberts, C. M.; Streyer, W.; Feng, K.; Fang, Z. Applied Physics Letters 2017, 111, 091105.

(20) Vassant, S.; Hugonin, J.-P.; Marquier, F.; Greffet, J.-J. Optics Express 2012, 20, 23971.

(21) Caldwell, J. D.; Lindsay, L.; Giannini, V.; Vurgaftman, I.; Reinecke, T. L.; Maier, S. A.; Glembocki, O. J. Nanophotonics 2015, 4, 44-68.

(22) Feng, K.; Streyer, W.; Zhong, Y.; Hoffman, A.; Wasserman, D. Optics Express 2015, 23, A1418.

(23) Caldwell, J. D.; Vurgaftman, I.; Tischler, J. G.; Glembocki, O. J.; Owrutsky, J. C.; Reinecke, T. L. Nature Nanotechnology 2016, 11, 9-15.

(24) Basov, D. N.; Fogler, M. M.; García De Abajo, F. J. Science 2016, 354.

(25) Low, T.; Chaves, A.; Caldwell, J. D.; Kumar, A.; Fang, N. X.; Avouris, P.; Heinz, T. F.; Guinea, F.; MartinMoreno, L.; Koppens, F. Nature Materials 2017, 16, 182-194.

(26) Simpkins, B. S.; Fears, K. P.; Dressick, W. J.; Spann, B. T.; Dunkelberger, A. D.; Owrutsky, J. C. ACS Photonics 2015, 2, 1460-1467.

(27) Dunkelberger, A. D.; Spann, B. T.; Fears, K. P.; Simpkins, B. S.; Owrutsky, J. C. Nature Communications 2016, 7. $1-10$.

(28) Burke, J. J.; Stegeman, G. I.; Tamir, T. Physical Review B 1986, 33, 5186-5201.

(29) Otto, A. Zeitschrift für Physik 1968, 216, 398-410.

(30) Passler, N. C.; Razdolski, I.; Gewinner, S.; Schöllkopf, W.; Wolf, M.; Paarmann, A. ACS Photonics 2017, 4, 1048-1053. 
(31) Hopfield, J. J. Physical Review 1958, 112, 1555-1567.

(32) Savona, V.; Hradil, Z.; Quattropani, A.; Schwendimann, P. Physical Review B 1994, 49, 8774-8779.

(33) Gubbin, C. R.; Martini, F.; Politi, A.; Maier, S. A.; De Liberato, S. Physical Review Letters 2016, 116, 1-6.

(34) Gubbin, C. R.; Maier, S. A.; De Liberato, S. Physical Review B 2016, 94, 1-9.

(35) Lidzey, D. G.; Bradley, D. D. C.; Skolnick, M. S.; Virgili, T.; Walker, S.; Whittaker, D. M. Nature 1998, 395, 53-55.

(36) Cade, N. I.; Ritman-Meer, T.; Richards, D. Physical Review B Condensed Matter and Materials Physics 2009, 79, 1-4.

(37) Baieva, S. V.; Hakala, T. K.; Toppari, J. J. Nanoscale research letters 2012, 7, 191.

(38) Lifshitz, E.; Brumer, M.; Kigel, A.; Sashchiuk, A.; Bashouti, M.; Sirota, M.; Galun, E.; Burshtein, Z.; Le Quang, A. Q.; Ledoux-Rak, I.; Zyss, J. The Journal of Physical Chemistry B 2006, 110, 2535625365.

(39) Huber, A. J.; Deutsch, B.; Novotny, L.; Hillenbrand, R. Applied Physics Letters 2008, 92, 2006-2009.

(40) Dai, S. et al. Nano Letters 2017, 17, 52855290.

(41) Schöllkopf, W.; Gewinner, S.; Junkes, H.; Paarmann, A.; von Helden, G.; Bluem, H.; Todd, A. M. M. 2015, 9512, 95121L.

(42) Passler, N. C.; Paarmann, A. Journal of the Optical Society of America B 2017, 34, 2128.

(43) Raether, H. Surface Plasmons on Smooth and Rough Surfaces and on Gratings; Springer Tracts in Modern Physics, 1988; Vol. 111.
(44) CST, CST Studio Suite. https://www. cst.com/products/csts2.

(45) Engelbrecht, F.; Helbig, R. Physical Review $B$ 1993, 48, 15698-15707.

(46) Moore, W. J.; Freitas, J. A.; Holm, R. T.; Kovalenkov, O.; Dmitriev, V. Applied Physics Letters 2005, 86, 1-3. 


\section{Graphical TOC Entry}

Some journals require a graphical entry for the Table of Contents. This should be laid out "print ready" so that the sizing of the text is correct. Inside the tocentry environment, the font used is Helvetica 8 pt, as required by Journal of the American Chemical Society.

The surrounding frame is $9 \mathrm{~cm}$ by $3.5 \mathrm{~cm}$, which is the maximum permitted for Journal of the American Chemical Society graphical table of content entries. The box will not resize if the content is too big: instead it will overflow the edge of the box.

This box and the associated title will always be printed on a separate page at the end of the document. 